\title{
The Effect of the Theorem Prover in Cognitive Science
}

\author{
Tadashi Takahashi ${ }^{1}$ and Hidetsune Kobayashi ${ }^{2}$ \\ 1 Dept. of Mathematics and Informatics, Faculty of Human Development, Kobe \\ University, 3-11, Tsurukabuto, Nada-ku, Kobe 657-8501, Japan \\ 2 Dept. of Mathematics, College of Science and Technology, Nihon University, 8-14, \\ Kanda-Surugadai 1-chome, Chiyoda-ku, Tokyo 101-8308, Japan
}

\begin{abstract}
Humans use strategies to solve problems. Strategies are used as knowledge to plan solutions and decide procedures. A computer algebra system with a theorem prover is being developed. We must consider the theorem prover from not only the perspective of its effect on cognitive science, but also from the perspective of mathematical studies.
\end{abstract}

\section{Introduction}

According to the three-level human behavior model of Rasmussen, automatic human actions can be classified into the three levels of skill-, rule- and knowledgebased actions ([6]). A skill-based action is a response that occurs in less than 1 second ([4]). A chain of skill-based actions is a rule-based action. Thinking about how to solve a problem is a knowledge-based action.

Skill-based actions are performed smoothly without intentional control. Rulebased actions require a great deal of repetitive practice in order to be transferred to the skill-based level. First, the external conditions must be recognized, then the rules for composing the act are combined with the conditions required to carry out the behavior. Knowledge-based actions require the recognition of external conditions, the interpretation of these conditions, the construction of a psychological model for considering solutions, planning, and finally, the use of the other two behavior levels to carry out the action. This is a process model in which mastery of behavior requiring thought is internalized to the point where it can be carried out unconsciously. Mistakes can be explained as omitted steps, or for example, as pushing the wrong nearby button in smoothly carried out skill-based actions. In the case of knowledge-based actions, illusion can lead to error. In the present study, this process was analyzed using Rasmussen's threelevel human behavior model in order to identify what functions are essential to facilitating smooth action and learning. Behavior used to learn about problems and how to solve them is classified in detail according to the three-level model. Humans act by classifying issues and their relationships by consciously combining them. Humans control themselves by constantly observing, thinking about, evaluating, and integrating their behavior in order to achieve accuracy, continuity, consistency, and normality ([3]). Classified factors can be separated into the same three levels as the general actions. 


\section{$2 \quad$ Strategy}

Strategies are used as knowledge to plan solutions and decide procedures. When these procedures, in general or for the most part, obtain the correct answer, the procedure is called a heuristic; however, such heuristics do not always result in a correct solution.

Strategies are used even when human beings solve mathematical problems. Recognition knowledge and experience are used as "doing it like this is effective in this case". The ability to rapidly reference knowledge is required for strategies based on experience. Furthermore, the recognition of thoughts and feelings controls. The famous book by the mathematician Polya, "How to solve it" $([5])$, showed the processes of mathematical problem solving; however, one can not learn how to use heuristics in problem solving just by reading a book. In researching problem solving, there are two contrasting concepts. The first emphasizes insight, flash, and senses, while the second emphasizes experiential knowledge. The former concept employs a strong tendency to perceive that strategies of thought are learned through the experience of problem solving. In other words, it is assumed that an intuitive feelings and specific technical abilities can be acquired. In the latter concept, it is assumed that problem solving ability arises from the accumulation of rules inherent to the domain provided by an individual problem. Such differences depend on the problem's nature, domain, and level, and the type of person involved in the learning process. In addition, it is difficult to establish clear boundary lines between these two concepts. In problem solving, experiential knowledge plays a large role. Heuristics are general ideas or algorithms (a procedure providing the correct solution), and are widely used. Heuristics are equal to "the logic of a thought". Examples of extremely general strategies are "try to draw a figure if you come across a difficult problem", and "search for similar problems that you have experience with". There are also concrete strategies we are familiar with, such as "A problem requiring the comparison of quantities requires two differences, and a transform formula" and "try to make clauses that differ next to each other for number sum sequence problems" ([1]).

\section{Theorem Prover}

As a representative of a theorem prover, the Isabelle/HOL system was used. Research on formalizing abstract algebra in Isabele/HOL is based on work by Hidetsune Kobayashi. This study focuses on researching mathematics, and in particular, on training researchers in the technics of proving ([2]). In the area of mechanical theorem proving, Kobayashi gave a decision procedure for what he called abstract algebra, based on algebraic method. It is really surprise to prove many abstract algebra theorems whose traditional proofs need enormous amounts of human intelligence. One of the key observations of Kobayashi is that theorems in abstract algebra can be relatively easily dealt with by a lot of lemmata, completely from former methods. The power of the method can be shown 
by experiments on computers in which many abstract algebra theorems were proved. The success of Kobayashi's method stimulated researchers to apply the connection of lemmata images. This research on formalizing abstract algebra in Isabelle/HOL is being conducted in order to develop a computer algebra system that supports mathematical study focused on abstract algebra. The system combines methods of automated theorem proving and also integrates programming in a natural way.

This method are of interest to researchers both in artificial intelligence (AI) and in algebraic modeling because they have been used in the design of programs that, in effect, can prove or disprove conjectured relationships between, or theorems about, abstract algebraic objects.

It is interesting to note that theorems have been verified by this method. In a limited sense, this "theorem prover" is capable of "reasoning" about algebraic conjectures, an area often considered to be solely the domain of human intelligence.

This research aims at extending current computer systems using facilities for supporting mathematical proving. The system consists of a general higherorder predicate logic prover and a collection of special provers. The individual provers imitate the proof style of human mathematicians and produce humanreadable proofs in natural language presented in nested cells. The long-term goal of this research is to produce a complete system, which supports mathematicians. On the meta-level, we can write explicit programs for reasoning tactics using Isabelle/HOL.

\section{Knowledge Base in Cognitive Science}

When researchers use the theorem prover for the acquisition of knowledge or skills, we must consider a "tool" to be a "symbol device". A symbol device exists between the researchers and the research subject. Operation activity occurs between a symbol device and the researching subject. In cognitive science, two difficulties exist, one in the interaction between the researcher and the symbol device, and one in the interaction between the symbol device and the research subject. Therefore, we must overcome these difficulties in order to effectively utilize the theorem prover in cognitive science. Moreover, we must assess the benefits of considering the integration of the theorem prover from the perspective of the relationship between mathematical knowledge and mathematical concepts. When theorem provers are used in mathematical studies, researchers achieve a result through their efforts. Then, the researchers must investigate whether conceptual problems exist or whether they simply do not appreciate how the theorem prover works. By using a theorem prover effectively, researchers become aware of numerous mathematical ideas. This is made possible by incorporating the results of research in cognitive science. In carrying out a seven-phase model of human action, "the formation of a series of intentions or actions" must be performed smoothly. The effective use of a theorem prover in cognitive science is influenced by the contents of mathematical thought, and research and understanding of 
mathematics can further influence general idea formation. The theorem prover influences the "perception - interpretation - evaluation" phases of evaluation. The foundations of this model were studied by Rasmussen as the three-level control model of individuals actions ([7]). We can use the theorem prover as a material object that is available for the assessment of human activity. The use of the theorem prover can establish automatic and routine procedures. Controlling this automation is essential, especially in research on though processes. There are three methods for creating a theorem proof (by hand, by mind, and with a computer). A researcher's point of view of cognitive science considers the relationship between the brain and mind as the relationship between hardware and software in a computer. According to this point of view, the science of the mind is a special science, the science of thought.

\section{Conclusion}

In the three-level model of human behavior, operations and strategies can be identified and considered in relation to human thought processes in order to facilitate error-free problem solving. In consideration of surface features and conditions, similar problems can be recognized and suitable problem-solving methods can be identified. In addition, it was found that contents of the subconscious can be raised to the knowledge-based action level in order to support the expression process and the achievement of efficient functioning.

The technology of theorem prover automated reasoning. The ultimate goal of mathematics is technology. To do mathematics is gaining knowledge and solving problems by reasoning. Theorem prover is a powerful tool for researching mathematics. Researchers should appreciate the possibility of sharing cognitive level with such technology.

\section{References}

1. Ichikawa S.: Psychology of Learning and Education, Iwanami Shoten, 91 (1995) (in Japanese).

2. Kobayashi H, Suzuki H and Ono Y.: Formalization of Henzels Lemma, 18th International Conference, TPHOLs, Oxford, UK, Emerging Trends Proceedings Oxford Research Report (2005).

3. Kozuya T. (ed.): Memory and Knowledge (Cognitive Psychology Lecture 2), University of Tokyo Press, 17 (1978) (in Japanese).

4. Polson P. G. and Kieras D. E.: A Quantitative Model of the Learning and Performance of Text Editing Knowledge, Proceedings of ACM CHI'85 Conference on Human Factors in Computing Systems. (1985).

5. Polya G.: How to solve it. Doubleday (1957).

6. Rasmussen J.: Recognition engineering of interface, Keigakushuppan (1990) (in Japanese).

7. Tamura H.: Human interface. Ohm-sha (1998) (in Japanese). 